\section{Ember: warming up to brown fat}

\section{By Michael J. Haas, Senior Writer}

A team led by Boston-area researchers has shown that inhibiting a cation channel dubbed TRPV4 induced white fat cells to behave like brown fat, thereby protecting mice from diet-induced obesity and insulin resistance. ${ }^{1}$ Ember Therapeutics Inc. has options to in-license the findings as a part of a trio of new deals for brown fat-related targets.

Brown adipose tissue (BAT), or brown fat, has a high mitochondrial content that allows it to burn and dissipate chemical energy as heat more efficiently than white adipose and other tissues. Although human adults have only small deposits of brown fat compared with infants and rodents, a potential strategy for treating obesity and related metabolic conditions is inducing white adipose tissue to take on the mitochondrial and thermogenic properties of brown fat-an approach known as browning.

Peroxisome proliferation-activated receptor- $\gamma$ coactivator $1 \alpha$ (PPARGC1A; PGC-1 $\alpha$ ) is a key player in oxidative metabolism and thermogenesis in multiple tissue types, ${ }^{2}$ and a previous study by the Boston team found that exposure to cold and other factors upregulated $P G C-1 \alpha$ in brown fat and skeletal muscle. ${ }^{3}$

Thus, the researchers postulated that compounds that induced $P G C-1 \alpha$ expression-directly or indirectly-could help treat metabolic diseases.

The team screened small molecule libraries for compounds that upregulated $P g c-1 \alpha$ in mouse white adipocytes and identified two hits that were known antagonists of transient receptor potential vanilloids (TRPVs), a subfamily of the transient receptor potential ion channels.

To determine if any of the six TRPVs known in humans and mice played a predominant role in activating $\operatorname{Pgc}-1 \alpha$, the team treated mouse white adipocytes with small hairpin RNAs against each of the four Trpvs targeted by the two hits-the other two Trpvs are not antagonized by these molecules. Knocking down Trpv4 (transient receptor potential vanilloid 4; VRL2) resulted in the greatest upregulation of $P g c-1 \alpha$ and other mitochondrial genes involved in thermogenesis.

In addition, Trpv4-deficient mice fed a high-fat diet gained less weight than wild-type controls on the same diet. The difference resulted from greater energy expenditure in the Trpv4-deficient models and not from changes in food intake, oxygen consumption or levels of physical activity.
The Trpv4-deficient models also had greater glucose tolerance and lower insulin resistance than controls.

In mouse models of diet-induced obesity, the TRPV4 antagonist GSK205 increased glucose tolerance and decreased insulin resistance compared with vehicle. The molecule did not produce weight loss, which the team attributed to poor pharmacokinetics. GSK205 is a research compound originally discovered by GlaxoSmithKline plc.

Additionally, because GSK205 is not orally available, it had to be given every day by intraperitoneal injection, team leader Bruce Spiegelman told SciBX. "The procedures involved put stress on the models and can cause them to lose weight anyway. So, although we did use proper controls in the experiment, we wouldn't have looked for GSK205 to induce weight loss."

Spiegelman is professor of cell biology and medicine at the DanaFarber Cancer Institute and Harvard Medical School. He also is a cofounder of Ember and chairman of its scientific advisory board.

The team included researchers from Boston Children's Hospital, Duke University School of Medicine, Howard Hughes Medical Institute, Massachusetts General Hospital and Scripps Florida.

Data were reported in Cell.

\section{Broadening the brown}

Ember, which did not fund any aspect of the Cell study, has secured an exclusive option from Dana-Farber to in-license the team's findings. The company thinks TRPV4 is a good target for inducing white fat to behave
- Lou Tartaglia, Therapeutics Inc. like brown fat, said Lou Tartaglia, president and interim CEO of Ember and partner at Third Rock Ventures.

The TRPV4 deal is one of a trio that Ember announced last week as part of an IP land grab in the brown fat space. The other deals were options to in-license IP for two other brown fat-related targets from Brigham and Women's Hospital-aldehyde dehydrogenase 1 family member A1 (ALDH1A1) and StAR-related lipid transfer domain containing 14 (STARD14; THEM1).

Studies in mouse models of obesity by separate teams at the institution showed that inhibiting Aldhlal induced brown fat-like attributes in white adipose tissue to limit weight gain and improve glucose homeostatis ${ }^{4,5}$ and that inhibiting Them1 increased fatty acid oxidation in brown fat to limit weight gain and induce resistance to diabetes, inflammation and hepatic steatosis. ${ }^{6}$

"All of the biology around these three targets indicates that inhibiting them browns fat and that all three are druggable," Tartaglia said.

The deals "reflect our review of activity in the brown fat space over the last nine months and our thinking that these three targets are the best of recent findings," he added.

He noted that blocking each target has a different mode of action in browning fat: inhibiting TRPV4 recruits brown fat by inducing brown fat-like gene expression and thermogenic properties in white fat; inhibiting ALDH1A1 activates brown fat-how is not clear-and also induces brown fat-like gene expression in white fat; and inhibiting 
THEM1 shunts fatty acid metabolism through brown fat.

Although these differences make it difficult to say which target might be the best to treat metabolic disease, the differences also "open up a possibility that inhibitors of two or all three could have additive effects on weight loss" and metabolic disease, Tartaglia told SciBX.

Ember's next step is to set up high throughput screens of small molecule libraries to identify lead inhibitors of the three targets and then develop the compounds to treat obesity and diabetes.

Given the in vivo results for GSK205 reported in the Cell paper, Tartaglia acknowledged that it is not yet clear whether TRPV4 inhibition could induce weight loss in already obese animals and thus potentially treat obesity in humans. "But we think the findings in the models of diet-induced obesity reflect the fact that the research compound GSK205 is far from optimal," he said. "We are not even using it as a starting point. Instead, we expect our screening efforts to identify more potent compounds that will have a more robust effect on weight loss."

Although the company's main focus is on biologics that target brown fat, "we are also fleshing out our pipeline because brown fat is such a new field," Tartaglia said. "It's not yet known whether a biologic or small molecule will make the better compound for targeting brown fat. So we're taking as broad an approach as possible to brown fat to thus identify the best pathway or target and type of therapeutic."

Ember has variants of the hormone irisin in preclinical testing to treat obesity. Irisin is a secreted form of fibronectin type III domain containing 5 (FNDC5) that plays a role in regulating brown fat development.

The company also is conducting target-based in vitro screens and pathway-oriented cellular screens for lead compounds that activate PR domain containing 16 (PRDM16) and forkhead box C2 (FOXC2; MFH-1) and biologics based on bone morphogenetic protein 7 (BMP7; OP-1).? All three proteins play roles in regulating the development of brown fat.

Dana-Farber has filed a patent application covering the findings reported in Cell.

Haas, M.J. SciBX 5(40); doi:10.1038/scibx.2012.1045

Published online Oct. 11, 2012

\section{REFERENCES}

1. Ye, L. et al. Cell; published online Sept. 28, 2012; doi:10.1016/j.cell.2012.08.034

Contact: Bruce M. Spiegelman, Dana-Farber Cancer Institute, Boston, Mass.

e-mail: bruce_spiegelman@dfci.harvard.edu

2. Puigserver, P. \& Spiegelman, B.M. Endocr. Rev. 24, 78-90 (2003)

3. Puigserver, P. et al. Cell 92, 829-839 (1998)

4. Kiefer, F.W. et al. Nat. Med. 18, 918-925 (2012)

5. Zhang, Y. et al. Proc. Natl. Acad. Sci. USA 109, 5417-5422 (2012)

6. Kotz, J. SciBX 5(22); doi:10.1038/scibx.2012.565

7. Rhodes, J. BioCentury 20(9), A16; Feb. 27, 2012

\section{COMPANIES AND INSTITUTIONS MENTIONED}

Boston Children's Hospital, Boston, Mass. Brigham and Women's Hospital, Boston, Mass. Ember Therapeutics Inc., Boston, Mass. Dana-Farber Cancer Institute, Boston, Mass. Duke University School of Medicine, Durham, N.C. GlaxoSmithKline plc (LSE:GSK; NYSE:GSK), London, U.K. Harvard Medical School, Boston, Mass.

Howard Hughes Medical Institute, Chevy Chase, Md. Massachusetts General Hospital, Boston, Mass.

Scripps Florida, Jupiter, Fla. Third Rock Ventures, Boston, Mass. 